\title{
On the Analytical Mechanics of Chemical Reactions. Quantum Mechanics of Linear Collisions*
}

\author{
R. A. Marcus \\ Noyes Chemical Laboratory, University of Illinois, Urbana, Illinois
}

(Received 3 June 1966)

\begin{abstract}
The analytical quantum mechanics of chemically reactive linear collisions is treated in the vibrationally near-adiabatic approximation. The "reaction coordinate" in this approximation is found to be the curve on which the classical local vibrational and internal centrifugal forces balance. Expressions are obtained for the calculation of transmission coefficients for these nonseparable systems. Some implications for tunneling calculations in the literature are noted. Expressions for nonadiabatic corrections are derived, the latter being associated with vibrational transitions undergone by the transmitted and reflected waves. When the system does not have enough energy to react, the last results refer to the vibration-translation energytransfer problem in linear collisions.

Two novel features are the introduction of an actual coordinate system which passes smoothly from one suited to reactants to one suited to products and the introduction of an adiabatic-separable method, a method which includes curvilinear effects. Extensions to collisions in higher dimensions are given in later papers.
\end{abstract}

\section{INTRODUCTION}

$\mathbf{I}^{\mathrm{N}}$ $\mathrm{N}$ the present paper the analytical quantum mechanics of chemically reactive linear collisions are examined. The resulting equations and the qualitative concepts can be compared with the results obtained ${ }^{1}$ by numerical integration of the Schrödinger equation. The equations are applied elsewhere to reaction tunneling problems and to a recently formulated ${ }^{2}$ statisticaldynamical theory for chemical reaction cross sections of $n$-dimensional systems. Comparisons with other studies are noted later. Elements in the present work stem from a recent series of investigations ${ }^{3,4}$ but it itself is self-contained.

For a linear collision only two coordinates in a center-of-mass system are needed to represent the pusitions of the atoms in a triatomic reaction, $\mathrm{A}+$ $\mathrm{BC} \rightarrow \mathrm{AB}+\mathrm{C}$. Unfortunately, the problem does not admit of separation of variables, except globally (or piecewise) for idealized potential-energy surfaces (sharp barriers etc.). ${ }^{5}$ Typically, the surface is plotted in skewed coordinates to eliminate cross terms in the kinetic energy. ${ }^{6}$ The surface normally possesses two valleys parallel to the axes, one corresponding to the initially separated reactants and the other to the finally separated products. The valleys are often separated by a saddle-point region (a "col"), and the

\footnotetext{
* Supported by a grant from the National Science Foundation.

${ }^{1}$ E. M. Mortensen and K. S. Pitzer, Chem. Soc. (London) Spec. Publ. 16, 57 (1962).

2 R. A. Marcus, J. Chem. Phys. 45, 2630 (1966).

3 R. A. Marcus, J. Chem. Phys. 41, 603, 610, 2614, 2624 (1964).

${ }^{4}$ R. A. Marcus, J. Chem. Phys, 43, 1598 (1965).

${ }^{5}$ For interesting studies made with that approximation, see H. M. Hulbert and J. O. Hirschfelder, J. Chem. Phys. 11, 276 (1943); D. W. Jepsen and J. O. Hirschfelder, ibid. 30, 1032 (1959); J. O. Hirschfelder and E. Wigner, ibid. 7, 616 (1939).

- S. Glasstone, K. J. Laidler, and H. Eyring, The Theory of Rate Processes (McGraw-Hill Book Co., Inc., New York, 1941), Chap. 3.
}

path of minimum potential energy leading up to the col from either valley is a curved one when projected onto the coordinate space. This minimum-potentialenergy path (ascent, descent) leading from reactants' valley to products' via intermediate configurations is called the "reaction path," to distinguish it from the "reaction coordinate," described later.

The vibrational motion of the system is initially a $\mathrm{BC}$ vibration, but, as one sees from the potential-energy contour plots in the above space changes its nature smoothly, becoming a symmetric stretching A $\cdots \mathrm{B} \cdots \mathrm{C}$ vibration in the saddle-point region and, finally, a vibration of the $\mathrm{AB}$ molecule. ${ }^{6}$ The system initially has a given vibrational quantum number for $\mathrm{BC}$. If there is a large probability of retaining this number in forming the product $A B$ the reaction can be called vibrationally adiabatic. Nonadiabatic transitions will occur when the system strikes the curved part of the reaction path too quickly or when the vibration frequency for motion perpendicular to the reaction path changes too rapidly along the path.

The quantum mechanics of these problems is considered here in the near vibrationally adiabatic limit. Nonadiabatic corrections are also calculated. It has been shown ${ }^{8}$ that some results of recent numerical

\footnotetext{
${ }^{7}$ H. S. Johnston, Advan. Chem. Phys. 3, 131 (1961).

${ }^{8}$ The classical results of Ref. 9 have been discussed briefly from this viewpoint in Ref. 4 while those in Ref. 10 have been considered in Ref. 2 and in a paper to be published. The validity of a zeroth-order vibrationally adiabatic approach is also evident from the relatively low transition probabilities fo other vibration states, as found in numerical quantum-mechanical studies (Ref. 1, Table I). The transmission coefficients in that study were analyzed in Ref. 3, p. 610, using an assumption which was tacitly vibrationally adiabatic. Some further examination of the latter is desirable utilizing the corrections for curvilinearity given in the present paper. Quantum adiabatic results concerning the role of zero-point energy were anticipated by L. S. Kassel, Kinetics of Homogeneous Gas Reactions (Chemical Catalog Company, New York, 1932), p. 59.
} 
studies $^{1,9,10}$ on the $\mathrm{H}+\mathrm{H}_{2} \rightarrow \mathrm{H}_{2}+\mathrm{H}$ reaction provide evidence that the reaction is adiabatic with respect to a certain vibration, at least at low to moderate relative velocities above threshold. The corresponding vibration in related three-center abstractions is also expected to be vibrationally adiabatic if the velocity of the moving point along the curved part of the reaction coordinate is not too high.

Vibrationally adiabatic reactions are of particular interest, since it has been shown ${ }^{11,12}$ that a thermal equilibrium distribution among activated-complex states is actually achieved for such reactions if the distribution of reactants ainong their own states is a thermal equilibrium one. That is, only for such reactions has a justification for activated-complex theory been given so far. ${ }^{13}$

The present calculations apply to the problem of vibrational energy transfer as well, for this case is a particular one in which the system does not have enough energy to react. Another closely related formulation for the latter problem, given elsewhere, ${ }^{14}$ is based less on the surface topography and more on the potential functions.

The two principal novel features of the present work and of its classical counterpart ${ }^{15}$ are the introduction of a coordinate system which passes very naturally from one appropriate to reactants to one appropriate to products, and the use of an adiabatic-separable approach in converting the partial differential equation (2) to ordinary differential equations.

\section{THEORY}

A coordinate system ${ }^{16}$ used also in a classical mechanical treatment ${ }^{15}$ of the problem is also used here. Let $C$ be a curve in the coordinate space. In some situations

${ }^{9}$ F. T. Wall, L. A. Hiller, Jr., and J. Mazur, J. Chem. Phys. 29,255 (1958).

${ }_{10}$ M. Karplus, R. N. Porter, and R. D. Sharma, J. Chem. Phys. 43, 3259 (1965).

${ }^{11} \mathrm{~J}$. O. Hirschfelder and E. Wigner, J. Chem. Phys. 7, 616 (1939); M. A. Eliason and J. O. Hirschfelder, ibid. 30, 1426 (1959).

${ }^{12}$ L. Hofacker, Z. Naturforsch. 18a, 607 (1963); cf. Ref. 4. ${ }_{13}$ While the adiabatic assumption may be a good approximation for formation of the symmetric stretching mode of $\mathrm{ABC}^{\ddagger}$, for dynamical reasons given in the present paper, it is less certain with regard to the bending modes of the activated complex (not discussed here). The latter arise from a combination of rotations of $\mathrm{BC}$ and orbital motion of $\mathrm{A}$ and $\mathrm{BC}$. Perhaps the best that can be hoped for dynamically is that the distribution of bending states is a random one about the adiabatic value. (See also Ref. 2.) A detailed analysis of existing electronic computer results will provide an answer to this problem.

${ }_{14}$ R. A. Marcus (to be published), where some comparison with the well-known method of Schwartz, Slawsky, and Herzfeld will be given.

${ }_{15}$ R. A. Marcus, J. Chem. Phys. 45, 4500 (1966), following paper.

${ }^{16} \mathrm{H}$. C. Corben and P. Stehle, Classical Mechanics (John Wiley \& Sons, Inc., New York, 1960), 2nd ed., pp. 319ff. For use of

(1) it is best that the system spend relatively little time in a certain region $D$ which is distant from $C$ on $C$ 's concave side. For, in Region $D$ the $x$-coordinate curves will eventually intersect, unless one modifies the coefficients in Eq. (1) in that region. Such intersections lead to difficulty. noted later it may be convenient to take $C$ to be the "reaction path." If $x$ denotes the shortest distance from any point near $C$ to $C$ and if $s$ denotes the distance along $C$ to that point, the position of a point in the space can be defined by specifying $x$ and $s$. The classical kinetic energy in this space is

$$
\mathrm{KE}=\frac{1}{2} \mu(1+\kappa x)^{2}(d s / d t)^{2}+\frac{1}{2} \mu(d x / d t)^{2} .
$$

In (1), $\kappa(s)$ is the curvature of $C$ at the particular $s$, and $\mu$ is the effective mass of the representative point describing the system. In mass-weighted space $\mu$ is unity, but in chemical kinetics calculations $\mu$ is usually chosen to equal the reduced mass of the two reactants. Only the units of the coordinates would be affected thereby, not the physical results.

In the present case, (as in Fig. 3 of Ref. 17 or Fig. 95-1 of Ref. 16) Curve $C$ is always concave to a singly connected region and convex to a second, and so the $\kappa$ in Eq. (1) is of constant sign. We choose it to be positive, thereby also choosing $x$ to be positive for points on the convex side of $C$.

In these curvilinear coordinates the Schrödinger equation ${ }^{18}$ is

$$
\left[-\frac{k^{2}}{\eta} \frac{\partial}{\partial s}\left(\frac{1}{\eta} \frac{\partial}{\partial s}\right)-\frac{k^{2}}{\eta} \frac{\partial}{\partial x}\left(\eta \frac{\partial}{\partial x}\right)+V\right] \Psi=E \Psi,
$$

where $k^{2}=\hbar^{2} / 2 \mu, V$ and $E$ are the potential and total energy, respectively, and

$$
\eta=1+\kappa x \text {. }
$$

$V$ is written as

$$
V=V_{1}(s)+V_{2}(x, s), \quad V_{2}(0, s)=0 .
$$

That is, $V_{1}(s)$ is the value of $V$ along $C$, and Eq. (4) serves as a definition for $V_{2}(x, s)$. If $C$ is chosen to be the "reaction path," $V_{2}$ is a Morse-like potential function of $x$ and $V_{1}(s)$ is the usual potential barrier function along that path, $V_{2}$ depends weakly on $s$.

A trial product solution of (2) is given by (5).19 Equations (4) and (5) are introduced into (2) and, after adding and subtracting $V_{1} / \eta^{2},(6)$ is obtained:

$$
\Psi(x, s)=\Psi^{(1)}(s, \alpha) \Psi^{(2)}(x, \alpha),
$$

where $\alpha$ denotes a constant $(E)$ and a quasiconstant $\left(\alpha_{2}\right.$ below) of the motion;

$$
\begin{aligned}
\frac{1}{\Psi^{(1)}}\left[-k^{2} \eta \frac{\partial}{\partial s}\left(\frac{1}{\eta} \frac{\partial}{\partial s}\right)+V_{1}(s)\right] \Psi^{(1)} \\
=\frac{-\eta^{2}}{\Psi^{(2)}}\left[\frac{-k^{2}}{\eta} \frac{\partial}{\partial x}\left(\eta \frac{\partial}{\partial x}\right)+U_{2}(x, s)-E\right] \Psi^{(2)}
\end{aligned}
$$

17 R. E. Weston, J. Chem. Phys. 31, 892 (1959).

18 For example, W. Pauli, Jr., in Handbuch der Physik, S. Flügge, Ed. (Springer-Verlag, Berlin, 1958), Vol. 5, p. 39. In our case $g^{8 s}$ is $1 / \mu(1+\kappa x)^{2}, g^{x x}$ is $1 / \mu$, and is $\mu(1+\kappa x)$, according to (1).

${ }^{19}$ This method is a less restricted version of the separableadiabatic one described in Ref. 4. For example, the condition made there on the kinetic-energy operator for the $s$ motion (present terminology) has been relaxed. 
where

$$
U_{2}(x, s)=\left\{1-\left[1 /(1+\kappa x)^{2}\right]\right\} V_{1}(s)+V_{2}(x, s) .
$$

For the moment we neglect $\partial \eta / \partial s$; in a region important for tunneling $\kappa$ is frequently constant and so $\partial \eta / \partial s=0$ there; outside that region $\kappa$ decreases smoothly to zero.

With the neglect of $\partial \eta / \partial s$, the left side of (6) depends only on $s$. The right side depends on $x$ and on $s$. If the reaction is largely vibrationally adiabatic, either the right side is only weakly dependent on $s$ or becomes so when a term $\beta(s)$ is subtracted from both sides of (6). Now both sides of (6) equal a constant therefore or equal a weakly s-dependent parameter. We absorb $\beta$ in this parameter and simplify the subsequent notation by writing the parameter as $\alpha_{2}+$ $\left(\frac{1}{2} \kappa k\right)^{2}$, yielding (8). The weak dependence of $\alpha_{2}$ on $s$ when Curve $C$ is chosen to coincide with the "reaction coordinate curve" is shown later by Eq. (18); typically, $\frac{1}{2}(\kappa k)^{2}$ is negligible:

$$
\begin{array}{r}
{\left[k^{2}\left(\partial^{2} / \partial s^{2}\right)+\alpha_{2}+\left(\frac{1}{2} \kappa k\right)^{2}-V_{1}\right] \Psi^{(1)}(s, \alpha)=0,} \\
{\left[\frac{k^{2}}{\eta} \frac{\partial}{\partial x}\left(\eta \frac{\partial}{\partial x}\right)+E-U_{2}(x, s)-\frac{1}{\eta^{2}}\left[\alpha_{2}+\left(\frac{1}{2} \kappa k\right)^{2}\right]\right]} \\
\times \Psi^{(2)}(x, \alpha)=0 .
\end{array}
$$

In $(9), \Psi^{(2)}$ is set equal to $\phi^{(2)} / \eta^{\frac{1}{k}}$, to give

$$
\begin{aligned}
\left\{-k^{2}\left(\partial^{2} / \partial x^{2}\right)+U_{2}(x, s)\right. & \left.+\left[\alpha_{2} /(1+\kappa x)^{2}\right]\right\} \\
& \times \phi^{(2)}(x, \alpha)=E \phi^{(2)}(x, \alpha) .
\end{aligned}
$$

The function $U_{2}(x, s)+\left[\alpha_{2} /(1+\kappa x)^{2}\right]$ serves as an effective potential-energy function for the vibrational motion, the second term and part of the first being of "an internal centrifugal" origin. The effective potential energy has a minimum at some $x, x_{0}$, which varies with $s$. Thereby, $x_{0}$ is defined by the equation ${ }^{20}$

$$
\partial V_{2}\left(x_{0}, s\right) / \partial x_{0}=\left(\alpha_{2}-V_{1}\right) 2 \kappa /\left(1+\kappa x_{0}\right)^{3} \text {. }
$$

This curve, $x=x_{0}(s)$, which acts as a center for the vibrational motion is the often-spoken-of "reaction coordinate." ${ }^{21}$ This coordinate was previously defined in the literature in the saddle-point region, neglecting all curvilinear effects, and in the regions of separated reactants and of separated products. ${ }^{6}$ It was not

${ }^{20}$ Throughout this paper $\partial V_{2}\left(x_{0}, s\right) / \partial x_{0}$ and $\partial^{2} V_{2}\left(x_{0}, s\right) / \partial x_{0}^{2}$ denote the values of $\partial V_{2}(x, s) / \partial x$ and $\partial^{2} V_{2}(x, s) / \partial x^{2}$ at $x=x_{0}$

${ }^{21} \mathrm{We}$ are using the term "reaction coordinate" in the sense that it has most frequently been used in the literature, namely as a coordinate along which tunneling rates may be estimated and which represents the vibrationally averaged position (harmonic-oscillator average, strictly speaking) as the system moves from reactants to activated complex to products. This particular coordinate curve is, of course, only a member of a family of coordinate curves, namely, curves of constant $x$ when $x_{0}$ is taken to be zero, but one having the vibrationally averaged and (for $x_{0}=0$ ) tunneling properties cited above. The families of coordinate curves, $x=$ constant and $s=$ constant, can be regarded as particular examples of the coordinate curves $q_{2}=$ constant and $q_{1}=$ constant in Ref. 4 . defined, however, in the regions in between. This lack prevented any dynamical discussion.

We denote by $\mathbf{H}^{x}$ an operator for the vibrational motion at any $s$,

$$
\begin{aligned}
\mathbf{H}^{x}=\frac{-k^{2}}{\eta} \frac{\partial}{\partial x}\left(\eta \frac{\partial}{\partial x}\right) & +U_{2}(x, s)-U_{2}\left(x_{0}, s\right) \\
& +\frac{\alpha_{2}+\left(\frac{1}{2} \kappa k\right)^{2}}{(1+\kappa x)^{2}}-\frac{\alpha_{2}}{\left(1+\kappa x_{0}\right)^{2}} .
\end{aligned}
$$

The vibrational eigenfunctions of $\mathbf{H}^{x}$ are denoted by $|n\rangle$ and the eigenvalues by $E_{n}$ (both $|n\rangle$ and $E_{n}$ vary with $s$ )

$$
\mathbf{H}^{x}|n\rangle=E_{n}|n\rangle \text {. }
$$

One sees by comparison of (9) with (12) and (13) that $|n\rangle$ is $\Psi^{(2)}(x, \alpha)$ and that

$$
E=E_{n}+U_{2}\left(x_{0}, s\right)+\left[\alpha_{2} /\left(1+\kappa x_{0}\right)^{2}\right] .
$$

Since $x_{0}$ depends only on $\alpha_{2}$ and $s$, as does $E_{n}$, Eqs. (11) and (14) serve to determine the functions $\alpha_{2}(s)$ and $x_{0}(s)$ for any specified $E$.

Equation (8) for the $s$ motion contains a kineticenergy term, a potential-energy term $V_{1}(s)$, a very small term $\left(\frac{1}{2} \kappa k\right)^{2}$, and $\alpha_{2}$ determined above. $\alpha_{2}$ is essentially the "local" energy for the $s$ motion referred to Curve $C$. This one-dimensional problem can be solved for the transmission coefficient and for $\Psi^{(1)}$ by standard techniques: numerical integration; simulation by a potential function which yields a solution in closed form; use of WBK or modified ${ }^{22} \mathrm{WBK}$ methods.

It is instructive to rewrite (14), using (8). We have

$$
\begin{aligned}
E=E_{n}+ & V_{1}(s)+V_{2}\left(x_{0}, s\right) \\
& -\left(\hbar^{2} / 2 \mu \Psi^{(1)} \eta_{0}^{2}\right)\left[\left(\partial^{2} / \partial s^{2}\right)+\left(\frac{1}{2} \kappa\right)^{2}\right] \Psi^{(1)} .
\end{aligned}
$$

The first term in (15) is the vibrational energy at any $s$; the second term is the potential energy at that $s$ on Curve $C$; the third term is the increment of potential energy on going from that point on $C$ to the point on the reaction coordinate for the same $s$. That is, the second and third terms constitute the potential energy on the reaction coordinate. The last term [exclusive of $\left(\frac{1}{2} \kappa\right)^{2}$ ] is the kinetic-energy contribution for the $s$ motion on the reaction coordinate. ${ }^{23}$ The term $\kappa^{2} \hbar^{2} / 8 \mu$

${ }^{22}$ The modified WBK method is found in J. Heading, $A n$ Introduction to Phase Integral Methods (Methuen and Co., Ltd., London, 1961), Chap. 5. The explicit equations are listed in Ref. 4, p. 1604. See also Eqs. (21.41) and (21.42) in E. C. Kemble, The Fundamental Principles of Quantum Mechanics (Dover Publications, Inc., New York, 1937), which anticipated the later results but which have not been noticed in the tunneling literature.

${ }_{23}$ (a) Incidentally, introduction of $\psi^{(1)}=\exp (i W / \hbar)$ into (15) yields $-p_{\mathrm{s}}^{2} / 2 \mu \eta_{0}{ }^{2}$ for the last term there, in the classical limit of $\hbar \rightarrow 0$, in the standard way. Here, $p$ is $d W / d s$ and is the momentum conjugate to $s$. (b) Introduction of $\psi^{(1)}=\exp (i W / \hbar)$ into (8) yields $-p_{\mathrm{s}}{ }^{2} / 2 \mu$ for the first term in brackets in (8) in the classical limit. The third term in brackets vanishes in that limit. Thus, the right side of (17) equals $p_{\mathrm{s}}{ }^{2} \kappa / \mu$ in this limit. It equals $\mu \dot{s}^{2} / R$, therefore, since $p_{\mathrm{s}}$ equals $\mu \dot{\mathrm{s}}\left(1+\kappa x_{0}\right)$, i.e., equals $\mu \dot{s}$ on $C$. $R$ is the local radius of curvature of $C, 1 / \kappa$, and $\mu \dot{s}^{2} / R$ is the wellknown centrifugal force. 
is normally very small and tends to zero in the classical limit.

The complete calculations are simplest though not the most accurate when $C$ is chosen to be the reaction path, since the dependence of $V$ on $x$ and $s$ is simplest then: $V_{2}(x, s)$ depends only on weakly $s$, and in the harmonic approximation $V$ is given by (16), where $\omega$ depends weakly on $s$ :

$$
V=V_{1}(s)+\frac{1}{2} \mu \omega^{2} x^{2}
$$

However, a term $1+\kappa x$ occurs in the adiabatic equation (9). When there is tunneling $x_{0}$ is negative and $1+\kappa x$ can get fairly small. $\alpha_{2}(s)$ then varies rapidly rather than weakly with $s$. In this situation, the choice of $x_{0}(s)=0$, instead of the reaction path, for Curve $C$ is much superior. In fact, since the $\kappa$ for such a curve is easily shown to be less than that for the reaction path in the tunneling region, $1+\kappa x$ turns out to be closer to unity for two reasons: (1) smaller $\kappa$, and (2) the typical $x$ is closer to zero since $x_{0}=0$.

When $C$ is chosen to correspond to $x_{0}=0, C$ is the reaction coordinate. In this case, Eqs. (11) and (14) become

$$
\begin{aligned}
\partial V_{2}\left(x_{0}, s\right) / \partial x_{0} & =\left(\alpha_{2}-V_{1}\right) 2 \kappa ; \quad x_{0}=0, \\
\alpha_{2} & =E-E_{n} .
\end{aligned}
$$

One can see from (8) that the right side of (17) is the local classical "internal centrifugal" force at the reaction coordinate. ${ }^{23 \mathrm{~b}}$ The left side is (except for sign) the local vibrational force there, at the same point. That is, the reaction coordinate is the curve for which these two forces balance.

\section{NONADIABATIC CORRECTION}

To apply standard formulas for perturbation or other theory, it is desirable that formally at least the unperturbed solution be an exact eigenfunction of some Hamiltonian. The solution of the preceding section is not. Rather, it is an approximate (adiabatic) solution. In this section, an unperturbed Hamiltonian $\mathrm{H}_{0}$ is derived for which the preceding solution [or, more precisely, one which agrees exactly with $\Psi^{(2)}(x, \alpha)$ and closely with $\left.\Psi^{(1)}(s, \alpha)\right]$ is an eigenfunction. Standard formulas are then applied.

In choosing $\mathbf{H}_{0}$ we proceed by analogy with a method given by Hofacker ${ }^{12}$ : Operators $\mathbf{H}_{(d)^{s}}$ and $\mathbf{H}_{(n d)}{ }^{s}$ are defined by analogy with his kinetic-energy operators, $\mathbf{T}_{(d)^{\xi}}$ and $\left.\mathbf{T}_{(n d)}\right)^{\text {. The }}$. The of a similar notation for the wavefunctions brings out some similarities.

We define a differential-integral operator $\mathbf{H}_{(d)}{ }^{s}$ by

$$
\mathbf{H}_{(d)^{8}}=\sum_{n}|n\rangle \mathbf{H}_{n n}\langle n|
$$

where the $|n\rangle$ form an orthormalized set (they are eigenfunctions of $\mathrm{H}^{x}$ ) and where

$$
\begin{aligned}
\mathbf{H}_{n n}= & \langle n|-\frac{k^{2}}{\eta} \frac{\partial}{\partial s}\left(\frac{1}{\eta} \frac{\partial}{\partial s}\right)-\frac{1}{\eta^{2}} \\
& \times\left[\alpha_{2}-V_{1}+\left(\frac{1}{2} \kappa k\right)^{2}\right]|n\rangle+U_{2}\left(x_{0}, s\right)+\alpha_{2} / \eta_{0}{ }^{2} .
\end{aligned}
$$

(See also Footnote 24.)

In (19) the integral operator $\langle n|$ is applied first, of course, before applying $\mathbf{H}_{n n}$. Thus, if $\mathbf{H}_{(d)}{ }^{8}$ operates on some $|m\rangle$ the $\partial / \partial s$ does not have an opportunity to differentiate that $|m\rangle$, since $\langle n \mid m\rangle=\delta_{m n}$. If $\mathbf{H}_{(d)}{ }^{8}$ operates on a product of $|m\rangle$ with some eigenfunction $|\widetilde{m} \nu\rangle$ below, the $\partial / \partial s$ [or what remains of it in the first term of (20)] does act on the latter function.

The eigenfunctions and eigenvalues of the operator $\mathbf{H}_{n n}+E_{n}$ are denoted by $|\tilde{n} \nu\rangle$ and by $E_{n \nu}$, respectively,

$$
\left(\mathrm{H}_{n n}+E_{n}\right)|\tilde{n} \nu\rangle=E_{n \nu}|\tilde{n} \nu\rangle .
$$

From these results it follows that a product function $|n \nu\rangle$, equal to $|n\rangle|\tilde{n} \nu\rangle$, is an eigenfunction of $\mathbf{H}_{0}$ with eigenvalue $E_{n \nu}$ :

where

$$
\mathrm{H}_{0}|n \nu\rangle=E_{n \nu}|n \nu\rangle
$$

$$
\left.\mathbf{H}_{0}=\mathbf{H}_{(d)}\right)^{s}+\mathbf{H}^{x} .
$$

An operator $H_{(n d)}{ }^{s}$ is next defined by

$$
\mathbf{H}_{(n d)^{s}}=\sum_{\substack{n, m \\ n \neq m}}|n\rangle \mathbf{H}_{n m}\langle m|,
$$

where $e^{24}$

$$
\begin{aligned}
\mathbf{H}_{n m}=\langle n|- & \frac{k^{2}}{\eta} \frac{\partial}{\partial s}\left(\frac{1}{\eta} \frac{\partial}{\partial s}\right)-\frac{1}{\eta^{2}} \\
& \times\left[\alpha_{2}-V_{1}+\left(\frac{1}{2} \kappa k\right)^{2}\right]|m\rangle, \quad n \neq m .
\end{aligned}
$$

It can be shown that the original Hamiltonian $\mathbf{H}$ is related to these operators by (26), i.e., that $\mathbf{H}_{(n d)}{ }^{8}$ can serve as the perturbation operator in standard formalisms,

$$
\mathbf{H}=\mathbf{H}_{0}+\mathbf{H}_{(n d)} \text {. }
$$

Various methods ${ }^{25}$ may be adapted to the present problem in order to determine transmission $(\kappa)$ and reflection ( $\rho$ ) coefficients. For example, in the Appendix

\footnotetext{
${ }^{24}$ Equations (20) and (25) could have been represented by a single equation:

that is, by

$$
H_{n m}=\left\langle n\left|\mathbf{H}-\mathbf{H}^{x}\right| m\right\rangle
$$

$H_{n m}=\langle n|-\left(k^{2} / \eta\right)(\partial / \partial s)\left[\eta^{-1}(\partial / \partial s)\right]-\left(1 / \eta^{2}\right)$

$\left.\times\left[\alpha_{2}-V_{1}+\left(\frac{1}{2} \kappa k\right)^{2}\right]+U_{2}\left(x_{0}, s\right)+\left(\alpha_{2} / \eta_{0}{ }^{2}\right) \mid m\right)$.

${ }^{25}$ For example, A. Messiah, Quantum Mechanics (Interscience Publishers, Inc., New York, 1963); T. Y. Wu and T. Ohmura, Quantum Theory of Scattering (Prentice-Hall Inc., Englewood Cliffs, N.J., 1962) ; N. F. Mott and H. S. W. Massey, The Theory of Atomic Collisions (Oxford University Press, Oxford, England, 1965), 3rd ed.
} 
it is shown that

$$
\begin{aligned}
& \kappa_{n \nu \rightarrow m \mu}=\left(v_{\infty}{ }^{m \mu} / v_{0}^{n \nu}\right) \\
& \quad \times\left|f_{n v, m \mu}{ }^{(+)}+\left(1 / i \hbar v_{c o}{ }^{m \mu}\right)\left\langle m \mu^{(-)}\left|\mathbf{H}_{(n d)^{s}}\right| \Psi_{n \nu}{ }^{(+)}\right\rangle\right|^{2}, \\
& \rho_{n \nu \rightarrow m \mu}=\left(v_{0}^{m \mu} / v_{0}^{n \nu}\right) \\
& \quad \times\left|f_{n v, m \mu}{ }^{(+)}-\left(1 / i \hbar v_{0}{ }^{m \mu}\right)\left\langle m \mu^{(-)}\left|\mathbf{H}_{(n d)^{s}}\right| \Psi_{n \nu}{ }^{(+)}\right\rangle\right|^{2},
\end{aligned}
$$

where $\Psi_{n \nu}{ }^{(+)}$is an eigenfunction of $\mathbf{H}$, incident from $s=-\infty$ in an initial state $|n \nu\rangle$ and consisting of incident plus outgoing waves. $\left|m_{\mu}^{(-)}\right\rangle$is an eigenfunction of $\mathbf{H}_{0}$ incident from $s=-\infty$ in (27) and from $s=+\infty$ in (28), in state $m \mu$, and consisting of incident plus incoming waves. The $v_{0}$ 's and $v_{\infty}$ 's are velocities at $s=-\infty$ at $s=+\infty$ for the states indicated by subscripts. The amplitude term $f_{n \nu, m \mu}{ }^{(+)}$occurs in the asymptotic value of an eigenfunction $\left|n \nu^{(t)}\right\rangle$ of the Hamiltonian $\mathbf{H}_{0}$. It describes transmission in (27) and reflection in (28), and vanishes unless $n \nu=m \mu$.

As a first approximation one could take $\Psi_{n \nu}{ }^{(+)} \cong$ $\left|n \nu^{(+)}\right\rangle$in (27) and (28), yielding

$$
\begin{aligned}
& \kappa_{n \nu \rightarrow m \mu}=\left(v_{\infty}^{m \mu} / v_{0}^{n \nu}\right) \\
& \quad \times\left|f_{n v, m \mu}^{(+)}+\left(1 / i h v_{\infty}{ }^{m \mu}\right)\left\langle\widetilde{m} \mu^{(-)}\left|\mathbf{H}_{m n}\right| \tilde{n}^{(+)}\right\rangle\right|^{2}, \\
& \rho_{n v \rightarrow m \mu}=\left(v_{0}^{m \mu} / v_{0}^{n \nu}\right) \\
& \quad \times\left|f_{n v, m \mu}^{(+)}+\left(1 / i h v_{\infty}{ }^{m \mu}\right)\left\langle\tilde{m}_{\mu}^{(-)}\left|\mathbf{H}_{m n}\right| \tilde{n}^{(+)}\right\rangle\right|^{2} .
\end{aligned}
$$

We conclude this section by considering the relation between the $|n \nu\rangle$ and the $\Psi^{(1)}$ of the preceding section. When $\partial \eta / \partial s$ is neglected and when derivatives of $|n\rangle$ with respect to $s$ are neglected, $\mathbf{H}_{n n}\left|\Psi^{(1)}\right\rangle$ yields (31) when $\mid \Psi^{(1)}$ is brought inside the integral in $\mathbf{H}_{n n}$ and (8) is introduced.

$\mathbf{H}_{n n}\left|\Psi^{(1)}\right\rangle=\left[U_{2}\left(x_{0}, s\right)+\alpha_{2} / \eta_{0}{ }^{2}\right]\left|\Psi^{(1)}\right\rangle=\left(E-E_{n}\right)\left|\Psi^{(1)}\right\rangle$,

where the second equality arises from (14). Comparison of (31) with (21) shows that $\left|\Psi^{(1)}\right\rangle$ is then the eigenfunction $|\tilde{n} \nu\rangle$ with eigenvalue $E_{n \nu}=E$, subject to the two approximations made in arriving at (31).

\section{HARMONIC-OSCILLATOR APPROXIMATION}

When the harmonic-oscillator approximation is introduced for the effective potential energy in (10) one finds

$$
E_{n}=\left(n+\frac{1}{2}\right) \hbar \omega_{2},
$$

where $n$ is the initial vibrational quantum number and $\mu \omega_{2}{ }^{2}=\left[\partial^{2} V_{2}\left(x_{0}, s\right) / \partial x_{0}^{2}\right]+\left[6\left(\alpha_{z}-V_{1}\right) \kappa^{2} /\left(1+\kappa x_{0}\right)^{4}\right]$.

When the reaction path is chosen as Curve $C$,

$$
\partial^{2} V_{2}\left(x_{0}, s\right) / \partial x_{0}{ }^{2}
$$

equals $\mu \omega^{2}$, where $\omega / 2 \pi$ is the "natural" frequency of vibration at $s$, i.e., the frequency if internal centrifugal forces were not considered [cf. Eq. (16)]. The value of $\omega / 2 \pi$ at the saddle point, $\omega_{\downarrow} / 2 \pi$, is the symmetric stretching vibration frequency there. When the reaction coordinate is Curve $C, x_{0} \equiv 0$ in (33).

For purposes of application to a tunneling problem in a later section, we need the equation for the relative position of the reaction coordinate and the reaction path at the barrier maximum when the former is chosen as Curve $C$. At the barrier maximum the normals to both curves coincide (exactly so, for symmetrical reactions at least). In this case let the path through the saddle point occur at $x=x_{t}, s=s_{t}$. Since the two normals coincide, $\partial^{2} V_{2} / \partial x^{2}$ at $x=0$ and $s=s_{\ddagger}$ equals $\partial^{2} V / \partial x^{2}$ at $x=0$ and $s=s_{\ddagger}$. Thereby, $V_{2}\left(x, s_{\ddagger}\right)$ is found to equal $\frac{1}{2}\left[\mu \omega_{\ddagger}^{2}\left(x-x_{\ddagger}\right)^{2}-\mu \omega_{\ddagger}^{2} x_{\ddagger}^{2}\right]$. Equation (17) then becomes

$$
-x_{\ddagger}=\left(\alpha_{2}-V_{1}\right) 2 \kappa / \mu \omega_{\ddagger}^{2}
$$

and $\alpha_{2}$ is given by (18). Let $V^{\ddagger}$ be the value of $V$ at the saddle point. Then $V_{1}$ in Eq. (34), the value of $V$ at the barrier maximum along $C$, is

$$
V_{1}=V^{\ddagger}+\frac{1}{2} \mu \omega_{\ddagger}^{2} x_{\ddagger}^{2} .
$$

\section{QUANTUM BOBSLED EFFECT}

The classical "bobsled" effect is one in which a system rushes headlong along a rectilinear path (downhill in the bobsled case), goes up one side of a valley when the valley bottom's path becomes curved, goes as far as it can, and continues downhill. This part of the effect can be largely adiabatic. When the initial translational velocity is very high, however, the bobsled not only goes up the valley wall as far as it can, but then goes down across the valley bottom, up the other side, and so on. This second effect involves induced oscillations where there were none initially. That is, it involves a vibrationally nonadiabatic effect, whose classical mechanics is treated analytically elsewhere. ${ }^{15}$ The adiabatic effect was observed by Wall and Porter ${ }^{26}$ in their electronic computer study of classical trajectories.

There is also an adiabatic quantum negative-positive centrifugal effect which could be mistaken for the nonadiabatic classical one. The former, which disappears when tunneling disappears, is seen from the preceding equations; when $\alpha_{2}<V_{1}$, i.e., when tunneling occurs, the classical velocity $\dot{s}$ becomes negative [Eq. (8)]. On taking Curve $C$ to be the reaction path $x_{\ddagger}$ equals zero. According to (11) with the left side equal to ${ }^{27}$ $\mu \omega^{2} x_{0}, x_{0}(s)$ is first zero (since $\kappa=0$ initially), then positive and then, if the system tunnels through the barrier, negative. Its negative property reflects the "negative kinetic energy" in the tunneling region, and was noted in an earlier paper on local approximation of a potential-energy surface by one permitting separation of variables. ${ }^{3}$

${ }^{26}$ F. T. Wall and R. N. Porter, J. Chem. Phys. 39, 3112 (1963). ${ }^{27}$ We write here the harmonic-oscillator expression for $\partial^{2} V_{2}\left(x_{0}, s\right) / \partial x_{0}^{2}$ for simplicity, but the subsequent argument applies as well to an anharmonic oscillator. 


\section{APPLICATION TO TUNNELING IN CHEMICAL REACTIONS}

In a WBK (or modified WBK) solution to the tunneling problem the integral

$$
\int_{a}^{b} p_{d} d s
$$

arises where $p_{s}^{2}$ is seen from Eq. (8) to equal $2 \mu\left[\alpha_{2}-V_{1}-\left(\frac{1}{2} \kappa k^{2}\right)\right]$ and where $a$ and $b$ are seen to be the points where $\alpha_{2}-V_{1}-\left(\frac{1}{2} \kappa k\right)^{2}$ vanishes. We neglect $\left(\frac{1}{2} \kappa k\right)^{2}$, a very small term $(\sim 0.2 \mathrm{kcal} / \mathrm{mole})$, for the moment. We choose the Curve $C$ so that $x_{0}(s)=0$ and use the harmonic-oscillator approximation for purposes of present discussion.

In the usual tunneling calculation one treats $E_{n}$ as constant, $\omega_{2}$ in (33) as $\omega_{t}$, and sets $\kappa=x_{\neq}=0$ at each $s$. In the curvilinear calculation we ignore, for purposes of present illustration, the variation in $\omega_{2}$ with $s$ in the interval $(a, b)$, and so write $\omega_{2}=\omega_{*}$. The tunneling rate, which depends on

$$
\int_{a}^{b} p_{s} d s
$$

is found to depend on the value of $\alpha_{2}-V_{1}$ at the barrier top. In the usual calculation, where $V_{1}$ is set equal to $V^{\ddagger}, \alpha_{2}-V_{1}$ is $E-E_{n}-V^{\ddagger}$, as one sees from (18). In the curvilinear calculation it is $E-E_{n}-V^{\ddagger}-\frac{1}{2} \mu \omega_{\ddagger}^{2} x_{\ddagger}^{2}$, [Eqs. (18) and (35)]. Thus, the difference in the two calculations occurs in the presence of a $\frac{1}{2} \mu \omega_{\ddagger}^{2} x_{\ddagger}^{2}$ term. [The turning points are also slightly different but we have ignored this, by taking $\omega_{2}(s) \cong \omega_{\ddagger}$, for purposes of the present illustration only.]

Some estimate of $\frac{1}{2} \mu \omega_{\ddagger}^{2} x_{\ddagger}^{2}$ is obtained for the $\mathrm{H}+\mathrm{H}_{2}$ reaction, as follows. For the Sato potential-energy surface used by Weston ${ }^{17}$ and by others, the curvature of the reaction path, $\kappa_{p}$, is about $3.4 \AA^{-1}$ in the neighborhood of the saddle point and $\omega_{\ddagger} / 2 \pi$ is $2108 \mathrm{~cm}^{-1}$. On taking $\kappa \simeq \kappa_{p}$ as a first approximation one finds $x_{4}=$ 0.054 and $0.108 \AA$, while $\frac{1}{2} \mu \omega_{\ddagger}^{2} x_{\ddagger}^{2}=0.37$ and 1.46 $\mathrm{kcal} / \mathrm{mole}$, when $\alpha_{2}-V_{1}=-2$ and $-4 \mathrm{kcal} / \mathrm{mole}$ at the saddle point. (An improved $\kappa$ could be calculated in each case using this $x_{\ddagger}$, but we omit this detail here.) Thus, the term controlling tunneling is -2 and $-4 \mathrm{kcal} / \mathrm{mole}$ now, but in the usual calculation it is -1.63 and -2.54 . The difference is appreciable and leads to less tunneling than those predicted by the usual calculations. The effect becomes increasingly marked the greater the negative centrifugal effect, i.e., the more negative $\alpha_{2}-V^{\ddagger}$ is.

It has been noted ${ }^{17}$ that tunneling rates predicted by the usual calculation are too high using the Sato surface. Smaller tunneling rates were calculated by Johnston and Rapp ${ }^{28}$ who considered tunneling through potential-energy profiles along straight-line sections parallel to the reaction path.

\footnotetext{
${ }^{28}$ H. S. Johnston and D. Rapp, J. Am. Chem. Soc. 83, 1 (1961).
}

\section{COMPARISON WITH OTHER STUDIES}

The nonadiabatic correction employed here may be compared with that developed by Hofacker ${ }^{12}$ in a very stimulating article. Hofacker assumes that a curvilinear coordinate system may be found, in terms of which a vibrationally adiabatic motion is simply described. In the nonadiabatic correction a purelykinetic-energy operator for motion along the reaction coordinate appeared, with elements $\mathbf{T}_{n n}$ instead of $\mathbf{H}_{n n}$, and elements $\mathbf{T}_{n m}$ instead of $\mathbf{H}_{n m}$. The two formalisms differ in that a method for obtaining the curvilinear coordinate system is obtained in the present paper. The article by Hofacker is more devoted to evolving a formalism for reaction-rate problems and did not attempt to determine the suitable coordinate system.

Other analytical approaches to reaction rate problems include those in which the system moves from the reactants' region to the products' one, along some valley having infinitely steep walls and having a curved shape permitting separation of variables throughout or piecewise. ${ }^{5}$ In the latter case nonadiabaticity occurs at the boundaries of the pieces.

Reference has already been made to the work of Johnston and $\operatorname{Rapp}^{28}$ on an intuitive approach to tunneling in the nonseparable region. In a quite different discussion, $R$-matrix theory has been used to describe sufficient, though not necessary, conditions for various reactive scattering models in the literature. ${ }^{29}$ A vibrationally adiabatic approach or some variant ${ }^{13}$ was not considered, it being more recent. ${ }^{2}$

An analytical approach to reaction-rate problems has recently been formulated using a distorted wave method. ${ }^{30}$ This method is normally used for inelastic events which have a low probability of occurrence. For example, one would expect it to be appropriate in linear collisions only if the transmission coefficient is low and only if the original choice for the distorted waves used is good. It will be interesting to compare the results of this method with the numerical ones and with those of the present approach.

\section{APPENDIX: DERIVATION OF EQS. (27) and (28)}

The present derivation does not exactly parallel one in the literature, since it is unusual that there is an approximate solution in the same coordinates leading smoothly from reactants to products. The arguments are rather similar to some ${ }^{31}$ in Ref. 25 , however.

Let $H$ and $\hat{H}$ be two Hamiltonians, with eigenfunctions $\Psi$ and $\hat{\Psi}$. Either $H$ or $\hat{H}$ or both may be $\mathbf{H}_{0}+\mathbf{H}_{(n d)^{8}}$ or $\mathbf{H}_{0}$. An eigenfunction in an incident state $a$ or $b$ is so indicated by a subscript. A (+) or $(-)$ superscript indicates incident plus outgoing and

${ }^{29}$ B. C. Eu and J. Ross, J. Chem. Phys. 44, 2467 (1966); see also E. E. Nikitin, Mol. Phys. 8, 473 (1964).

${ }^{30}$ D. Micha, Arkiv Fysik 30, 411, 425, 437 (1965).

${ }^{31}$ A. Messiah, Ref. 24, pp. 836-839. 
incident plus incoming wave, respectively. A wave may be incident from $s=-\infty$ or $s=+\infty$. Let $\Psi_{a}^{(+)}$be incident from $s=-\infty$ and $\Psi_{b}{ }^{(-)}$incident from $s=-\infty$ or $+\infty$, as indicated.

Integration by parts, using the configuration space volume element $\eta d x d s$, yield (A1) :

$$
\begin{aligned}
& \left\langle\hat{\Psi}_{b}^{(-)}|H-\hat{H}| \Psi_{a}^{(+)}\right\rangle \\
& =-\left.\frac{\hbar^{2}}{2 \mu} \int_{x=-\infty}^{+\infty}\left(\Psi_{a}^{(+)} \frac{\partial}{\partial s} \hat{\Psi}_{b}^{(-) *}-\hat{\Psi}_{b}(-) * \frac{\partial \Psi_{a}^{(+)}}{\partial s}\right) d x\right|_{s=-\infty} ^{+\infty},
\end{aligned}
$$

the right side being the indicated difference of limits at $s=+\infty$ and $s=-\infty$, respectively.

Let $\phi_{\gamma}$ be a vibrational eigenfunction of $H$ or $\hat{H}$ at $s= \pm \infty$. It is the same for the cited choices of $H$ and $\hat{H}$, but its value at $s=+\infty$ may differ from that at $s=-\infty$. The asymptotic behavior of $\Psi_{a}^{(+)}$is

$$
\begin{aligned}
\Psi_{a}^{(+)} & \underset{s \rightarrow-\infty}{\sim} \phi_{a} \exp \left(i k^{a} s\right)+\sum_{\gamma} \phi_{\gamma} g_{a \gamma}{ }^{(+)} \exp \left(-i k^{\gamma} s\right) \\
& \sim \sum \phi_{\gamma} g_{a \gamma}{ }^{(+)} \exp \left(i k^{\gamma} s\right)
\end{aligned}
$$

since at large $|s|$ the eigenfunctions become plane waves (linear combinations of). $[\kappa \rightarrow 0$ (and so $\eta \rightarrow 1$ ) in Eq. (2) in the regions of separated reactants $(s=-\infty)$ and separated products $(s=+\infty)$.] In (A2) $k^{a}, k^{\gamma}$, and $g_{a \gamma}{ }^{(+)}$should bear subscripts of $-\infty$ to indicate that they refer to their values at $s=-\infty$. In (A3) they should bear subscripts $+\infty$. For brevity, these symbols are omitted at first. The $k$ 's are wavenumbers $\left(p_{s} / \hbar\right)$.

Two choices for $\Psi_{b}^{(-)}$are considered, permitting the determination of $g_{a b}(+)$ at $s=-\infty$ and at $s=+\infty$, respectively, (1) $\hat{\Psi}_{b}^{(-)}$incident from $s=-\infty$,

$$
\begin{aligned}
\hat{\Psi}_{b}^{(-)} & \underset{s \rightarrow-\infty}{\sim} \sum_{\gamma} \phi_{\gamma} \hat{g}_{b \gamma}(\rightarrow) \exp \left(i k^{\gamma} s\right) \\
& \sim \phi_{b} \exp \left(i k^{b} s\right)+\sum_{\gamma} \phi_{\gamma} \hat{g}_{b \gamma}(-) \exp \left(-i k^{\gamma} s\right)
\end{aligned}
$$

and $(2) \hat{\Psi}_{b}(-)$ incident from $s=+\infty$,

$$
\begin{aligned}
\hat{\Psi}_{b}^{(-)} & \sim \phi_{b} \exp \left(-i k^{b} s\right)+\sum_{\gamma} \phi_{\gamma} \hat{g}_{b \gamma}^{(-)} \exp \left(i k^{\gamma} s\right) \\
& \sim \sum_{s \rightarrow+\infty} \phi_{\gamma} \hat{g}_{b \gamma}{ }^{(-)} \exp \left(-i k^{\gamma} s\right) .
\end{aligned}
$$

On introducing (A2) to (A5) in (A1), (A8) is obtained, while (A9) is obtained by introducing instead (A2), (A3), (A6), and (A7):

$$
\begin{aligned}
& \left\langle\hat{\Psi}_{b}^{(-)}|H-\hat{H}| \Psi_{a}^{(+)}\right\rangle \\
& \quad=i \hbar\left[v_{\infty}{ }^{b} g_{a b}(+)(\infty)-v_{-\infty}{ }^{a} \widehat{g}_{b a}^{(-) *}(-\infty)\right], \\
& \left\langle\hat{\Psi}_{b}^{(-)}|H-\hat{H}| \Psi_{a}^{+}\right\rangle \\
& =-i \hbar\left[v_{-\infty}^{b} g_{a b}^{(+)}(-\infty)-v_{-\infty}^{a} \widehat{g}_{b a}^{(-) *}(-\infty)\right],
\end{aligned}
$$

where $g_{a b}+( \pm \infty)$ is the value of $g_{a b}{ }^{+}$at $s= \pm \infty$ and $v=p_{s} / \mu . v_{-\infty}^{b}$, for example, is the value of $p_{s} / \mu$ for a system in State $b$ and at $s=-\infty$.

Choice of $H=\widehat{H}$ yields

and

$$
v_{\infty}^{b} g_{a b}(+)(\infty)=v_{-\infty}{ }^{a} g_{b a L}(-) *(-\infty)
$$

$$
v_{-\infty}{ }^{b} g_{a b}{ }^{(+)}(-\infty)=v_{-\infty}{ }^{a} g_{b a R}(-)(-\infty),
$$

where $L$ indicates that $\hat{\Psi}_{b}(-)$ is incident from the left (i.e., from $s=-\infty$ ) and $R$ indicates its incidence from $s=+\infty$. Choice of $H=\mathbf{H}_{0}+\mathbf{H}_{(n d)}{ }^{s}$ and $\hat{H}=\mathbf{H}_{0}$ yields Eqs. (27) and (28), when use is made of the results just obtained and when one notes that $\kappa_{a \rightarrow b}$ is

$$
v_{\infty}^{b}\left|g_{a b}+(\infty)\right|^{2} / v_{-\infty} a .
$$

In Eqs. (27) to (30) the $-\infty$ subscripts have been replaced by 0 's to conform with previous notation. 